\title{
NANOSTRUCTURED LIPID CARRIERS (NLCS): A PROMINENT TOPICAL DELIVERY SYSTEM FOR COENZYME Q10 AND MYRICA ESCULENTA LEAVES EXTRACT FOR ANTI-AGING POTENTIAL
}

\author{
PRASHANT KUMAR, ANITA SINGH* \\ Department of Pharmaceutical Sciences, Kumaun University Bhimtal, Uttarakhand, India 263136 \\ Email: dranitaku@gmail.com
}

Received: 05 Jun 2018 Revised and Accepted: 14 Jul 2018

ABSTRACT

Objective: In this study, Myrica esculenta (ME) leaves extract along with Coenzyme Q10 was entrapped in nanostructured lipid carriers (NLCs). The formulation variables were successfully optimized using $2^{3}$ factorial design.

Methods: Preparation of NLC was carried out by solvent injection technique. The formulation of NLC was consisting of the methanolic extract of $M y r i c a$ esculenta leaves and Coenzyme Q10 as key ingredients. Oleic Acid (OA) was taken as liquid lipid, Precirol ATO 5 (PATO) as solid lipid, and Poloxamer 407 (P407). The formulation variables were statically optimized using $2^{3}$ factorial design, followed by the characterization of the formulation.

Results: The results demonstrated that the best optimized NLC formulation F5 of Coenzyme Q10 and plant extract exhibited the smallest size $169.46 \mathrm{~nm}$ and highest entrapment efficiency $90.06 \pm 0.171 \%$. Furthermore, it showed good physical stability at $28 \mathrm{~d}$ after preparation.

Conclusion: The developed NLCs of Q10-ME extract from this study could be explored as the effective anti-ageing formulation.

Keywords: Anti-aging cosmetics, Antioxidant activity, Factorial design nanostructured lipid carriers

(C) 2018 The Authors. Published by Innovare Academic Sciences Pvt Ltd. This is an open access article under the CC BY license (http://creativecommons.org/licenses/by/4.0/) DOI: http://dx.doi.org/10.22159/ijpps.2018v10i8.27745

\section{INTRODUCTION}

The human being of the modern world had a great inclination to search for an everlasting youth. In this physical world facial appearance became a crucial factor to influence social relations in many situations due to which people tend to judge personality based on facial manifestation [1]. This desire had ultimately triggered for the development of new products, methods and technology in the cosmeceutical industry which might revert back the clock [2]. Skin is the largest human organ contributing, a major target of oxidative stress [3]. Skin aging is a gradual deterioration of the physiological functions of skin which results in wrinkles or loose skin that causes unattractive appearances. Several theories have been laid down to describe the mechanism of the skin aging of which the most successful and referred is the free radical theory based on oxidative stress [4].

Many antioxidant agents have been studied to develop novel antiaging products. Coenzyme Q10 one of the most popular antioxidants, naturally occurring in the skin that had been studied in this field [5] most common products composed of CoQ10 generally contained in the range of $0.5-1.0 \%$ in anti-wrinkle products as well as in antioxidant products. It is having the tendency to show the adverse effect at higher doses if taken orally [6].

To solve this problem lipid-based nano-particulate drug carriers have been developed to increase solubility and improve the bioavailability of poorly water soluble and/or lipophilic drugs [7]. The plant extracts usually contain polyphenols such as flavonoids, which react with reactive oxygen species (ROS) to neutralize the harmful effects of free radicals [8].

Myrica esculenta Buch.-Ham. (Myricaceae) commonly known as Box Berry, Kaiphal, and Katphala in Ayurveda is widely used treatment of gulma (abdominal tumors), jvara (fever), arsa (piles), grahani (irregular bowel function), pandu roga (anemia), hrillasa (nausea), mukha roga. The medicinal properties of M. Esculenta are well known as per ethnobotanical surveys. Therefore in present study researcher had made an attempt to develop topical drug delivery system which may enhance the potency of Coenzyme Q10 by incorporating methanolic extract of $\mathrm{M}$. esculenta into NLC carriers to offer a great potential to justify the objectives of present work [9]. Considering these aspects, researchers have selected NLCs as carriers due to their special nanostructures which tend to improved properties for therapeutic loading, better drug release profile and stability [10].

\section{MATERIALS AND METHODS}

Coenzyme Q10 (CoQ10) was purchased from Sigma-Aldrich Chemicals, St. Louis, MO. Precirol ATO 5 (PATO), Oleic Acid (OA) and Poloxamer 407 (P407) were provided as gift samples from Gattefossé Saint-Priest, France and BASF Corporation, USA respectively. All other chemicals and reagents used were of analytical grade and procured from SD Fine Chem Limited, Mumbai. The plant specimen, i.e. dried leaves of M. esculenta were collected from the northern Himalayan region of Garhwal Uttarakhand, India in the month of March 2015 from the height of 1685.53 Meters N $29.8688^{\circ} \mathrm{N}, 78.8383^{\circ} \mathrm{E}$. The plants were identified and authenticated in BSI, Allahabad, India (voucher specimen BSA94627). The Standardization of plant extract was carried out through HPLC method followed by quantification of gallic acid in M. esculenta extract using RP-HPLC. The NLCs were prepared through the solvent injection technique and statically optimized by $2^{3}$ factorial design [11-12].

\section{Standardization of plant extract through HPLC method}

M. esculenta methanolic extract was standardized through reversephase high-performance liquid chromatography (RP-HPLC). Lyophilized hydro-alcoholic extract of $M$. esculenta was accurately weighted and volume of the solution was adjusted in a volumetric flask to obtain a final concentration of $1 \mathrm{mg} / \mathrm{ml}$. The optimized mobile phase consisted of acetone with $1 \%$ acetic acid in water in the ratio of 76: $24(\mathrm{v} / \mathrm{v})$ with the flow rate of $1 \mathrm{ml} / \mathrm{min}$. The HPLC system comprised of rheodyne-7725 injection valve with a sample loop (20 ml), Vacuum degasser, quaternary pump and photo-diode array detector (PDA) was used, with data acquisition by Empower TM 2 software (Waters 600, Milford, MA, USA). Chromatography was performed on a Spherisorb C18 column $(250 \mathrm{~mm} \times 4.6 \mathrm{~mm}, 5 \mathrm{~mm}$; Waters, Ireland) fitted with a C18 guard column $(10 \times 3.0 \mathrm{~mm})$. The sample elution was performed at $25{ }^{\circ} \mathrm{C}$ and detected at the UV 
wavelength of $254 \mathrm{~nm}$. A sample was analyzed by HPLC using $20 \mu \mathrm{L}$ injection volume of a syringe (Hamilton Microliters; Switzerland). The $\mathrm{pH}$ meter (Orion 3 Star, Thermo-Scientific) was used to adjust the $\mathrm{pH}$ of the mobile phase. Amount of the marker compound present in the crude plant extract was determined through the calibration curve. Calibration curve constructed by the corresponding peak area of the gallic acid as standard was plotted against the concentration of standards by means of linear regression. The stock solution of a standard compound was prepared by dissolving the known amount of gallic acid in methanol at a concentration of $1 \mathrm{mg} / \mathrm{ml}$.

\section{Quantification of gallic acid in M. esculenta extract}

M. esculenta extract was standardized by RP-HPLC under the isocratic conditions using the external standard calibration technique. A bioactive compound was identified by comparing with the respective retention time (Rt) of the gallic acid as standard. The calibration curve was plotted by plotting peak areas against concentrations and five standard marker ranges from 100-500 $\mu \mathrm{g} / \mathrm{ml}$. The standard compound shows a good linearity between concentrations and the peak area, with the correlation coefficient $\left(\mathrm{r}^{2}\right)$ of 0.998 .

Obtained chromatograms from HPLC analyses of the extract and their respective markers are shown in fig. 1. The optimum separation of was achieved by using the mobile system at the volume ratio of acetone with $1 \%$ acetic acid in water in the ratio of $74: 24(\mathrm{v} / \mathrm{v})$ with a flow rate of $1 \mathrm{ml} / \mathrm{min}$. The retention time of gallic acid was found to be $4.013 \mathrm{~min}$. The percentage amount of standard constituent (gallic acid) present in the crude extract was found at $1.64 \%(\mathrm{w} / \mathrm{w})$.
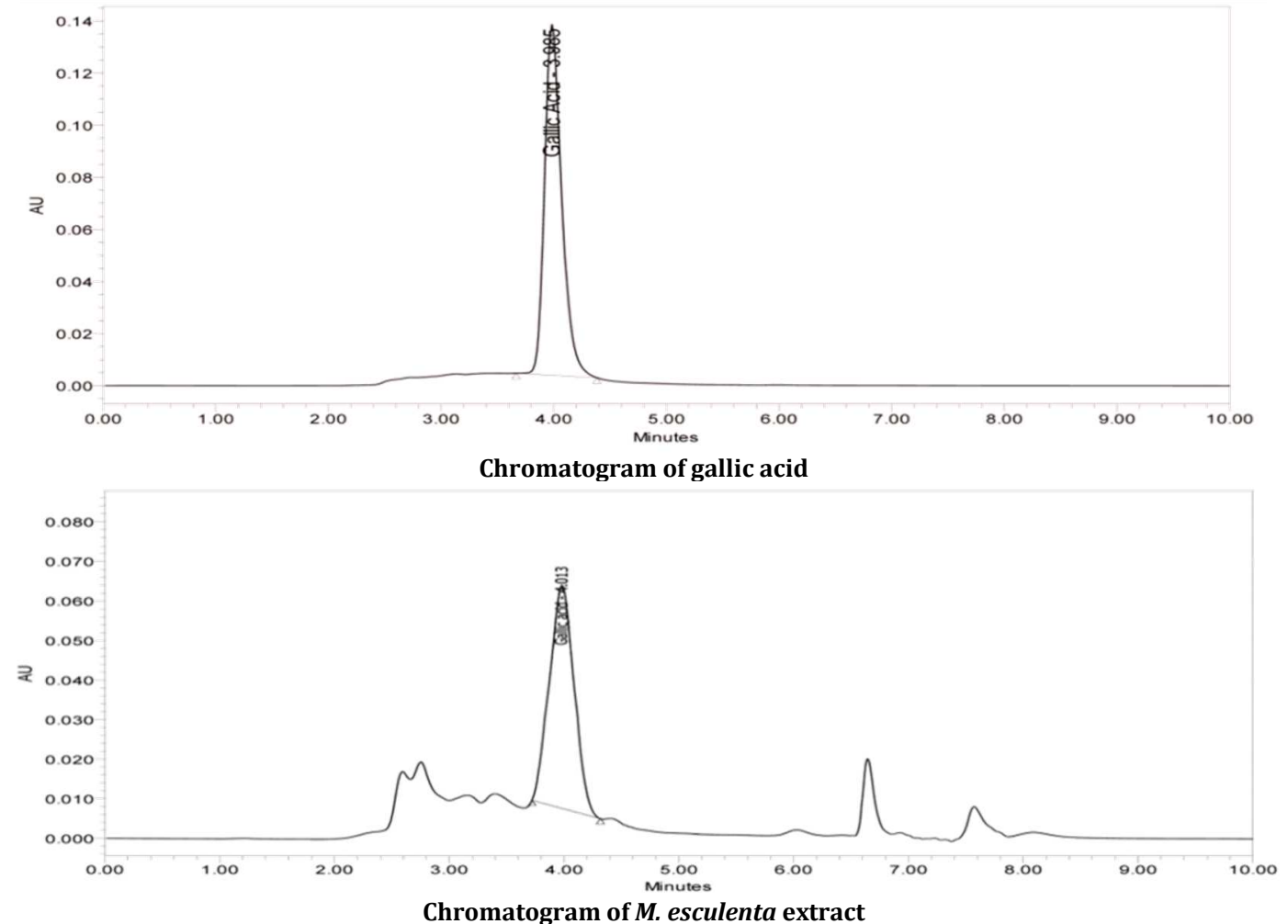

Fig. 1: RP-HPLC chromatograms of gallic acid and M. esculenta extract

\section{Excipient compatibility test}

Excipient compatibility test was carried out for $21 \mathrm{~d}$ by taking the physical mixture in 1:5 ratio of CoQ10 separately with PATO, OA and P407 respectively at temperature $40 \pm 2{ }^{\circ} \mathrm{C}$ and relative humidity $75 \pm 5 \%$.

\section{Experimental design of NLCs}

In this study, $2^{3}$ factorial design was used to optimize NLCs. In order to optimize, the amount of solid lipid, (PATO, $\mathrm{X}_{1}$ ), amount of liquid lipid $\left(\mathrm{OA}, \mathrm{X}_{2}\right)$ and concentration of surfactant $\left(\mathrm{P} 407, \mathrm{X}_{3}\right)$ were selected as independent variables. Each factor was set at two levels +1 and -1 . The actual values and coded values of different variables are given in table 1. Eight formulations of NLCs (F1-F8) were prepared according to design. The particle size and \% entrapment efficiency were taken as response parameters.

\section{Evaluation of response parameters of NLC}

\section{Particle size and zeta potential}

The particle size and the zeta potential of the formulations were measured using Malvern Zetasizer. The polydispersity index (PDI) was used as a parameter of the size distribution. The samples were diluted with double distilled water prior to particle size and zeta potential determination at $25^{\circ} \mathrm{C}$.

\section{Entrapment efficiency}

The percent entrapment efficiency of the CoQ10-ME loaded NLC was calculated by using the equation reported by Shah and Pathak, 2010 [13] and \%EE was calculated by the following equation:

$$
\% \mathrm{EE}=[\mathrm{Wa}-(\mathrm{Ws}+\mathrm{Wp}) / \mathrm{Wa}]
$$

Where Wa is the amount of drug added in the system, Ws is the amount of drug in the supernatant after centrifugation, and $\mathrm{Wp}$ is the amount of drug in the purification medium.

\section{Drug content determination by HPLC}

Estimation of gallic acid (GA) content in the formulations was analyzed by HPLC. (The standard stock solution was prepared by dissolving the known amount of GA in methanol to get a concentration of $1000 \mu \mathrm{g} / \mathrm{ml}$. Different aliquots were prepared by subsequent dilution with the same solvent. Samples were stored at 4 ${ }^{\circ} \mathrm{C}$ and protected from light before using. In this analysis, the mobile phase of acetonitrile and deionized water was used as a proportion 
of 35:65, 30:70 and 25:75 v/v (1\% glacial acetic acid; $\mathrm{pH} 2.2)$ which were sonicated for $15 \mathrm{~min}$ and degassed prior to use. Before applying all samples were also filtered through $0.45 \mu \mathrm{m}$ (NYL) syringe filter. The recoveries of standard compounds from various samples and formulation at three concentration levels were determined by measuring the percentages of detecting concentrations over additional concentrations. The quantitative analysis was repeated about three times $(n=3)$ by comparing and interpolating the peak area (response) of the sample with the standard.

Table 1: Formulation of NLC of $Q_{10}$-ME using $2^{3}$ factorial design

\begin{tabular}{|c|c|c|c|c|c|}
\hline $\begin{array}{l}\text { Formulation } \\
\text { code }\end{array}$ & $\begin{array}{l}\text { Amount of CoQ10 and plant extract } \\
(1: 1)\end{array}$ & $\begin{array}{l}\text { Amount of pato } \\
\text { (X1) }\end{array}$ & $\begin{array}{l}\text { Amount of } \\
\text { OA (X2) }\end{array}$ & $\begin{array}{l}\text { Amount of } \\
\text { P407 (X3) }\end{array}$ & $\begin{array}{l}\text { Response } \\
\text { parameter }\end{array}$ \\
\hline F1 & 10 & -1 & -1 & -1 & Particle size \\
\hline $\mathrm{F} 2$ & 10 & +1 & -1 & -1 & percentage \\
\hline F3 & 10 & -1 & +1 & -1 & entrapment efficiency \\
\hline $\mathrm{F} 4$ & 10 & -1 & -1 & +1 & \\
\hline F5 & 10 & +1 & +1 & -1 & \\
\hline F6 & 10 & +1 & -1 & +1 & \\
\hline F7 & 10 & -1 & +1 & +1 & \\
\hline F8 & 10 & +1 & +1 & +1 & \\
\hline F9 & 10 & -0.098 & -0.834 & +0.187 & \\
\hline
\end{tabular}

Where: $\mathrm{X} 1:-1=0.5 \%$ and $+1=2.5 \% \mathrm{w} / \mathrm{w}: \mathrm{X} 2:-1=0.10 \%$ and $+1=0.30 \% \mathrm{w} / \mathrm{w}: \mathrm{X} 3:-1=0.80 \%$ and $+1=1.2 \% \mathrm{w} / \mathrm{v}$

\section{Statistical analysis}

The effect of independent variables on the responses was modeled by Design Expert software version 11 (Stat-Ease, Inc., Minneapolis, USA). Polynomial equations were generated for the dependent variables that were reduced by removing non-significant coefficients by applying one way ANOVA. Level of significance was set at $\mathrm{p}<0.05$ [14].

\section{Validation of experimental design}

The experimental design was validated by an extra checkpoint formulation (F9) [15]. The predicted values for particle size and entrapment efficiency for $\mathrm{F}$, generated by their respective polynomial equations were compared with experimental values and tested for statistical significance using pooled t-test at $95 \%$ confidence interval, and degree of freedom $=3(p<0.05)[16]$.

\section{Selection of the optimized formulation}

Optimized formulation was selected on the basis of the desirability factor as calculated by the Design Expert software version 11 (StatEase, Inc., Minneapolis, USA).

\section{Visualization by transmission electron microscopy}

The morphology of selected CoQ10 ME loaded NLC dispersion was observed by transmission electron microscopy. One drop of the CoQ10 ME loaded NLC dispersion was spread on a carbon-coated copper grid and the excess droplets were removed with filter paper.
A drop of $4 \% \mathrm{w} / \mathrm{v}$ phosphotungstic acid solution was dropped into the grid. The negatively stained sample was air dried at room temperature and examined at 15000X magnification.

\section{Stability studies}

Stability of the selected optimized NLCs dispersion was determined as per ICH guidelines for 28days Effects of temperature and relative humidity on physical changes, particle size, and drug content was studied for NLC dispersion [17].

\section{RESULTS}

\section{Drug-excipient compatibility}

Drug-excipient compatibility was determined by the binary mixture method. After $21 \mathrm{~d}$ no physical change was observed. The drug content was found out to be $97.25,96.46$ and $98.62 \%$ for physical mixture PATO, $\mathrm{OA}$ and P407 respectively. Thus it could be said that the excipients selected for the formulation are safe and have no impact on the efficacy of the product. Furthermore, it is supported with the help of interaction plots obtained by the statistical design $\left(2^{3}\right.$ factorial design).

\section{$2^{3}$ Factorial design}

The factorial design was used for optimization of CoQ10-ME loaded NLC as it is an effective tool for optimization of independent variables. Thus, formulation F1-F8 were prepared according to the factorial design and evaluated for the dependent variables.

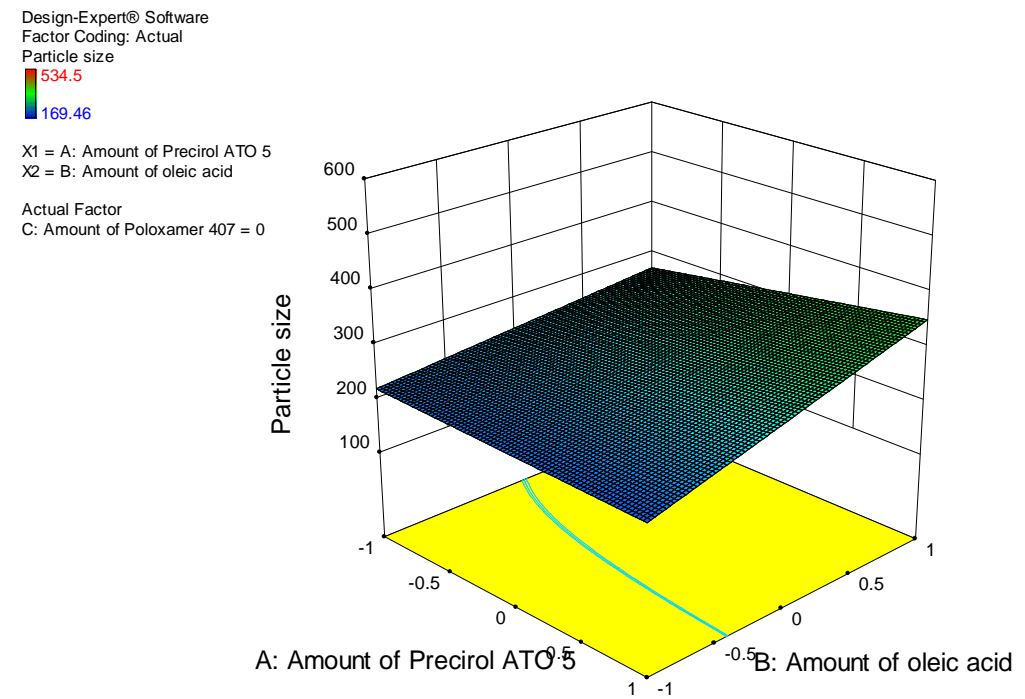

Fig. 2: Response surface plot for particle size analysis 


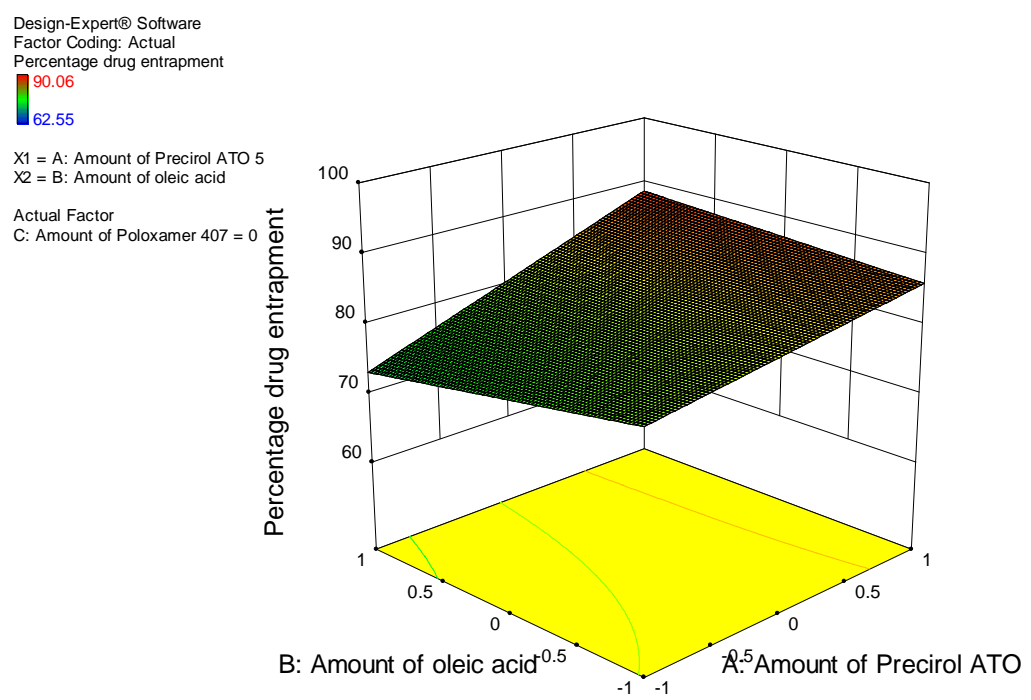

Fig. 3: Response surface plot for analysis entrapment efficiency

\section{Preparation of nanostructured lipid carriers}

NLCs were prepared by solvent injection technique that is based on rapid diffusion of the solvent across the solvent-lipid interface with the aqueous phase [18]. Thus, the diffusion rate of the organic solvent through the interface seems to be a critical parameter for the particle size determination. The major limitation with NLCs is its separation due to their small size and low density of the lipids. To overcome this limitation, in the present study, the $\mathrm{pH}$ of the dispersion was reduced to 1.5-2 to adjust the zeta potential for aggregation of NLCs and facilitate the centrifugation and consequently separation.

\section{Particle size and zeta potential measurements}

Values of the particle size and surface charge of the developed NLCs are shown in table 2 . The results showed that the amounts of PATO and $\mathrm{OA}$ were critical parameters governing the particle size. After production, the mean diameters of the particles were in the range of $165.9 \sim 226.8 \mathrm{~nm}$, depending on the lipid loading, and polydispersity was $<0.45$. Upon increasing the $0 A$ percentage in the lipid matrix from $0.10 \%$ to $0.30 \% \mathrm{w} / \mathrm{w}$, the mean particle size decreased. $\mathrm{F} 5$ exhibited the smallest size among all the formulations tested. As depicted in table 2, the zeta potential value of the lipid nanoparticles was negative.

\section{Drug entrapment efficiency}

An important issue with respect to the use of nanoparticles as drug carriers is their capacity for drug loading. The effects of $\mathrm{OA}$ concentration on drug entrapment efficiency of NLC were investigated. It is clear that the drug entrapment efficiency of nanoparticles increased from 80.63 to $94.50 \%$ respectively, with increasing the percentage of OA from 0.1 to $0.3 \%$ by weight (table 2).

Table 2: Physical characterization of CoQ10-ME NLCs

\begin{tabular}{llll}
\hline Formulation code & Particle size (nm) & PDI $^{\mathbf{a}}$ & Zeta potentiala $^{\mathbf{a}}(\mathbf{m V})$ \\
\hline F1 & $176.52 \pm 0.12$ & $0.243 \pm 0.09$ & $-30.01 \pm 0.31$ \\
F2 & $214.18 \pm 0.31$ & $0.261 \pm 0.21$ & $-31.39 \pm 0.52$ \\
F3 & $288.57 \pm 0.65$ & $0.263 \pm 0.10$ & $-30.02 \pm 0.46$ \\
F4 & $264.58 \pm 017$ & $0.267 \pm 0.74$ & $-28.08 \pm 0.17$ \\
F5 & $169.46 \pm 0.58$ & $0.212 \pm 0.17$ & $-36.39 \pm 0.08$ \\
F6 & $197.27 \pm 0.09$ & $0.249 \pm 0.45$ & $-31.89 \pm 0.24$ \\
F7 & $254.2 \pm 0.16$ & $0.243 \pm 0.76$ & $-33.45 \pm 0.78$ \\
F8 & $534.5 \pm 0.27$ & $0.384 \pm 0.08$ & $-28.51 \pm 0.09$ \\
F9(P) & $226.25 \pm 0.10$ & $0.267 \pm 0.14$ & $-31.28 \pm 0.11$ \\
F9(E) & $226.11 \pm 0.29$ & $0.258 \pm 0.35$ & $-31.35 \pm 0.34$ \\
\hline
\end{tabular}

$\mathrm{P}=$ Predicted, $\mathrm{E}=$ Estimated, $\mathrm{a}=$ mean $\pm \mathrm{SD}, \mathrm{n}=3$.

\section{Statistical analysis}

Statistical analysis was done by Design expert software version 11 (Stat-Ease, Inc., Minneapolis, USA) and the second order polynomial equations were derived. The transformed equations are,

$$
\begin{aligned}
\text { Particle size }=264.41 & +16.41 \mathrm{X} 1+49.27 \mathrm{X} 2+50.23 \mathrm{X} 3 \\
& +23.86 \mathrm{X} 1 \mathrm{X} 2+36.81 \mathrm{X} 1 \mathrm{X} 3+32.44 \mathrm{X} 2 \mathrm{X} 3 \\
& +63.05 \mathrm{X} 1 \mathrm{X} 2 \mathrm{X} 3
\end{aligned}
$$

Percentage drug entrapment

$$
\begin{aligned}
& =82.04+5.34 \mathrm{X} 1-1.03 \mathrm{X} 2+2.34 \mathrm{X} 3 \\
& +2.40 \mathrm{X} 1 \mathrm{X} 2-2.78 \mathrm{X} 2 \mathrm{X} 3+2.36 \mathrm{X} 1 \mathrm{X} 3 \\
& -3.24 \mathrm{X} 1 \mathrm{X} 2 \mathrm{X} 3
\end{aligned}
$$

Where X1, X2, and X3 are the independent variables. The effects of various independent variables on the dependent variables are illustrated in fig. 2 and fig. 3 that clearly depicts the effect of varying the levels of independent factors on dependent factors.

These polynomial equations were utilized for validation of the experimental design. An extra design checkpoint formulation (F9) was prepared. Calculation of the predicted value(s) for particle size and \% EE were made and compared with the experimental values as determined by evaluating F9. Close resemblance between predicted and experimental values indicated the validity of the generated model.

\section{Assessment of interaction in independent variables}

The possible excipient interaction was analyzed with the help of graphs obtained by the statistical design, for each response parameter(s), which in turn indicates the significance of experimental design (fig. 4 and fig. 5) 

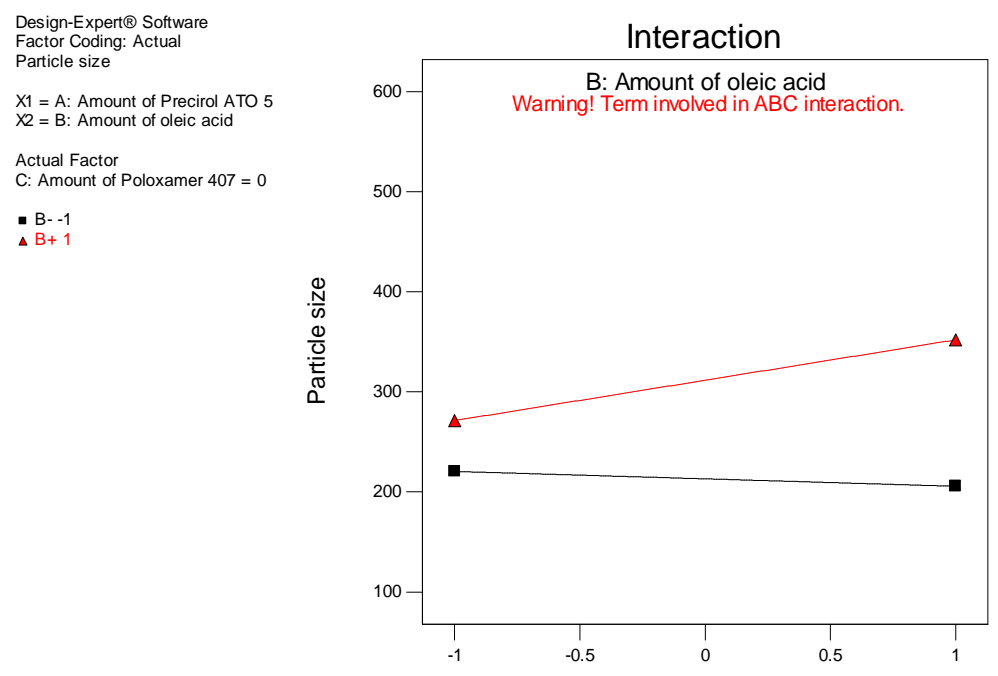

A: Amount of Precirol ATO 5

Fig. 4: Interaction between independent factors and particle size
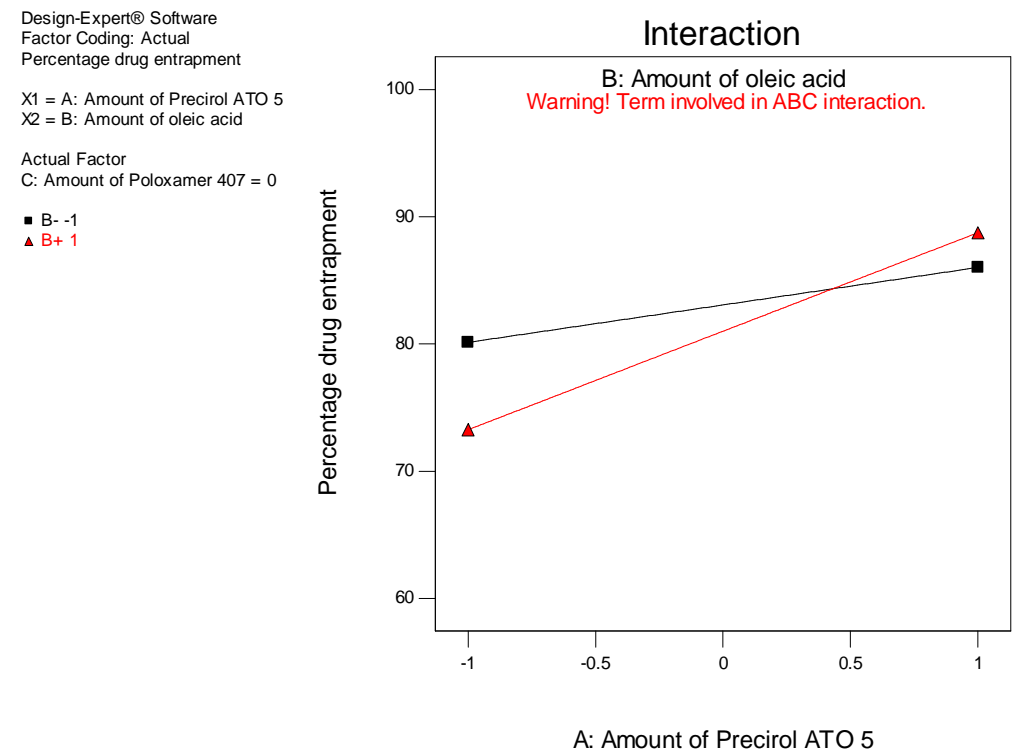

Fig. 5: Interaction between independent factors and entrapment efficiency

\section{Validation of the experimental design}

An extra design checkpoint formulation (F9) was made and the predicted value and experimental values of dependent variables were compared using pooled t-test at $95 \%$ confidence interval, a degree of freedom 3 and $\mathrm{p}<0.05$. No significant difference was recorded between the two values (table 2) thereby establishing the validity of the generated model.

\section{Selection of optimized formulation}

Eventually, F9 with desirability factor $=1$, the particle size of 226.11 $\mathrm{nm}$ and entrapment efficiency of $82.55 \pm 0.41 \%$, was identified as the optimized formulation.

\section{Transmission electron microscopy}

Transmission electron microscopic image of NLC indicated spherically shaped particles with narrow size distribution. No aggregation was observed as shown in fig. 3.8. As viewed, all the particles were less than $200 \mathrm{~nm}$, a size particularly suitable for topical delivery.

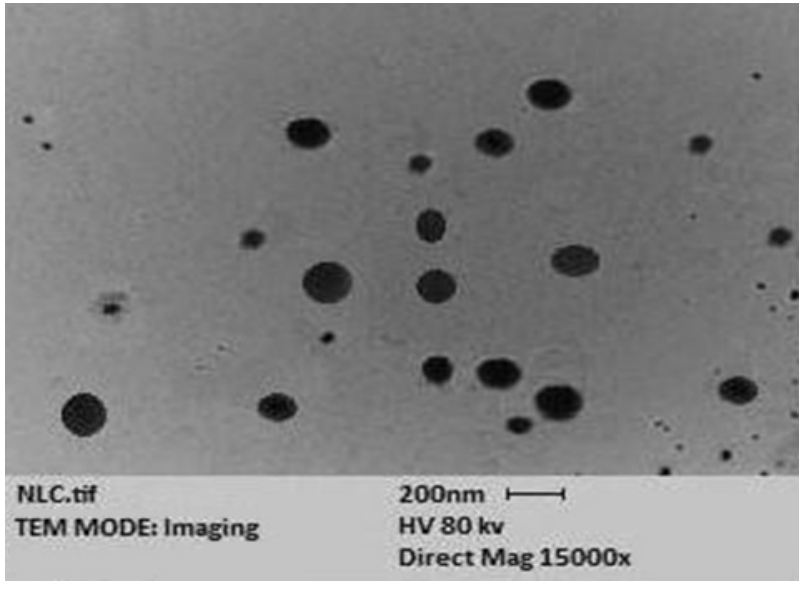

Fig. 6: Transmission electron microscopy of NLC formulation F5 


\section{Stability studies}

Stability of the selected optimized NLCs dispersion was determined as per ICH guidelines on the basis of effects of temperature and relative humidity on physical changes, particle size, and drug content was studied for NLC dispersion. The drug content found out to be $98.16 \%$, 95.49 and $93.38 \%$ on subjecting the formulation for $28 \mathrm{~d}$ at $25 \pm 2{ }^{\circ} \mathrm{C}$ temperature $60 \pm 5 \% \mathrm{RH}, 30 \pm 2{ }^{\circ} \mathrm{C}$ temperature $65 \pm 5 \% \mathrm{RH}$ and $40 \pm 2{ }^{\circ} \mathrm{C}$ temperature $75 \pm 5 \% \mathrm{RH}$ respectively. At the end of the study, there was no any sign of physical change or deformity.

\section{DISCUSSION}

The results of the experimental design were analyzed using Design Expert software. The selected independent variables like the amount of PATO, amount of $\mathrm{OA}$ and concentration of P407 had significantly influenced the particle size and, \% EE that is very much evident from the polynomial equations. The possible interactions between X1X2, X2X3, and X1X3 for each response were also investigated (fig. 4 and fig. 5).

Graphically, the interactions are visualized by lack of parallelism in the lines, for each response parameter(s) indicated interactions. The response surface plots (fig. 2 and fig. 3 ) generated using polynomial equations represent a simultaneous effect of any two variables on response parameter taking one variable at a constant level. On carefully observing these plots, the qualitative effect of each variable on each response parameter can be visualized.

\section{The effect of each independent variable was observed on these} dependent variables and the following conclusions were drawn

In increasing the amount of PATO, the particle size increased, indicating that the particle size is highly dependent on the amount of lipid used. On increasing the lipid content the viscosity of the lipidsolvent phase increase resulting in the reduction in the diffusion rate of the solute molecules to the outer phase that results in an increase in particle size. But as observed in table 2 the increase in particle size leveled at $2 \% \mathrm{w} / \mathrm{w}$ of PATO and on further increase in the amount of PATO, the particle size decreased.

The abundance of lipid results in diffusion of lipid to the outer core of NLC resulting in a decrease in particle size. In addition to particle size, the lipid content also affected the entrapment efficiency. An increase in the amount of PATO resulted in an increase in \% EE increase, higher Precirol content means availability of more amounts of the triglycerides containing a binary mixture of tripalmitin and tristearin, act as solubilizing agents for the lipophilic drug(s) [19].

Careful analysis of the experimental design revealed that the liquid lipid, oleic acid as another major component that affected the particle size of NLCs. As indicated by factorial design, the particle size decreased while moving from lower level to higher level of OA. Thus when the amount of OA increased from-1 level to +1 level, the particle size decreased from $534.5 \mathrm{~nm}$ to $169.46 \mathrm{~nm}$.

The third independent variable, P407 was also varied at different levels using factorial design and its effect on particle size was analyzed. At the lowest level, the particle size was $214.18 \mathrm{~nm}$ (formulation F2) that dropped to $197.27 \mathrm{~nm}$ (formulation F6) at the highest level of P407 indicating that increase in the amount of poloxamer can cause a decrease in particle size.

Increase in P407 causes a surfactant induced reduction in surface tension and prevents aggregation of the particles consequently leading to a decrease in particle size. Similarly, the percent entrapment efficiency was observed to decrease from $90.06 \pm 0.171$ $\%$ to $87.43 \pm 0.359 \%$ as we moved from -1 to +1 level of surfactants.

According to our criteria of lower particle size, higher \% EE, and higher desirability factor, F9 was selected as optimized formulation (desirability value of 1.0). Consequently, the coded optimized level for the amount of PATO, amount of OA, the concentration of P407, were identified as $-0.098,-0.834$, and +0.187 , respectively. These coded optimized values can be converted to actual optimized values by using principles of transformation by use of the following equation [20].

Coded Value $(\mathrm{x})=\{$ Actual value of $\mathrm{X}$ - Average of the actual level $\}$ / /0.5difference between the actual level
Transmission electron microscopic image of optimized NLC indicated spherically shaped particles with narrow size distribution. No aggregation was observed as shown in fig. 6 .

\section{CONCLUSION}

Nanostructured lipid carrier of Coenzyme $Q_{10}$ and $M$. esculenta leaves extract were successfully developed and statistically optimized by using $2^{3}$ factorial design. The obtained NLCs were nanosized particles with high entrapment efficiency. The enhanced permeation of the drug through NLC carriers might help in enhancing the pharmacological efficacy of $\mathrm{CoQ}_{10}$, subsequently enhancing the antiaging potential of $\mathrm{CoQ}_{10}$ with polyphenolic herbal extract of $M$. esculenta leaves. The statistically optimized CoQ10-ME NLC carriers containing GA may prove the prominent and enhanced antiaging potential of CoQ10.

\section{ACKNOWLEDGEMENT}

The authors are thankful to the Department of Pharmaceutical Sciences, Faculty of Technology, Kumaun University, Bhimtal, Nainital-263136, Uttarakhand for providing all the facilities to conduct this research work.

\section{AUTHORS CONTRIBUTIONS}

All the author have contributed equally

\section{CONFLICT OF INTERESTS}

The authors declare no conflict of interest

\section{REFERENCES}

1. Makrantonaki E, Zouboulis CC. Skin alterations and diseases in advanced age. Drug Discovery Today Dis Mech 2008;5:53-62.

2. Ramos-e-Silva M, Celem LR, Ramos-e-Silva S, Fucci-da-Costa AP. Anti-aging cosmetics: facts and controversies. Clin Dermatol 2013;31:750-8.

3. Kohen R, Gati I. Skin low molecular weight antioxidants and their role in aging and in oxidative stress. Toxicology 2000;148:149-57.

4. Junqueira VB, Barros SB, Chan SS, Rodrigues L, Giavarotti L, Abud RL, et al. Aging and oxidative stress. Mol Aspects Med 2005;25:5-16.

5. Mukul S, Surabhi K, Atul N. Cosmeceuticals for the skin: an overview. Asian J Pharm Clin Res 2017;4:16.

6. P Kaur, Kapila M, Agrawal R. Role of novel delivery systems in developing topical antioxidants as therapeutics to combat photoaging, Ageing Res Rev 2007;6:271-88.

7. Gardouh AR, Samar HF, Ahmed TN, Ghorab MM. Influence of formulation factors on the size of nanostructured lipid carriers and nanoemulsions prepared by high shear homogenization. Int J Pharm Pharm Sci 2018;10:61-75.

8. O'Driscoll CM, Griffin BT. Biopharmaceutical challenges associated with drugs with low aqueous solubility-the potential impact of lipid-based formulations. Adv Drug Delivery Rev 2008;60:617-24.

9. Pamudji JS, Mauldin R, Indrani N. Development of NLC formulation containing retinyl palmitate. Int J Pharm Pharm Sci 2016;8:256-60.

10. Kumar P, Chabra G, Tiwari A, Pathak K. Use of central composite design for statistical optimization promethazine teoclate loaded solid lipid nanoparticles. Asian J Pharm 2014;8:279-86.

11. Kumar VR, Gouda TS, Sreelakshmi S, Rajasekar. Phytochemical analysis and in vitro antioxidant activity of Ochna obtusata. Int J Pharm Res Scholars 2014;3:211-6.

12. Niamprem P, Srinivas SP, Tiyaboonchai W. Development and characterization of indomethacin-loaded mucoadhesive nanostructured lipid carriers for topical ocular delivery. Int J Appl Pharm 2018;10:91-9.

13. Shah M, Pathak K. Development and statistical optimization of solid lipid nanoparticles of simvastatin by using $2^{3}$ full factorial design. AAPS Pharm Sci Tech 2010;11:489-95.

14. Yue Y, Zhou H, Liu G, Li Y, Yan Z, Duan M. The advantages of a novel CoQ10 delivery system in skin photoprotection. Int J Pharm 2010;392:57-63.

15. Shaikh AC, Qazi A, Nazim S. Formulation optimization of hydrodynamically balanced oral controlled Release 
bioadhesive tablets of tramadol hydrochloride. Asian J Pharm Clin Res 2011;4:61-70.

16. Srivastava R, Abdul MA, Sharma A, Kumar P, Upadhyay G, Anwar F. Validation of a statistically optimized stability indicating the method for the estimation of febuxostat in a solid dosage form. EC Pharm Sci 2015;1:133-47.

17. ICH Guidelines Q1; 2008.

18. Keshri L, Pathak K. Development of thermodynamically stable NLC system using the central composite design for zero order permeation of econazole nitrate through the epidermis. Pharm Dev Technol 2014;18:634-44.

19. Doktorovova S, Araujo J, Garcia ML, Rakovsky E, Souto EB. Formulating fluticasone propionate in novel PEG-containing nanostructured lipid carriers (PEG-NLC). Colloids Surf B 2010;75:538-42.

20. Souto EB, Wissing SA, Barbosa CM, Muller RH. Development of a controlled release formulation based on SLN and NLC for topical clotrimazole delivery. Int J Pharm 2004;271:71-7. 